\title{
EI arte urbano como patrimonio inmaterial. Posibilidades para su protección y difusión
}

\section{Laura Luque Rodrigo ${ }^{a}$ y Carmen Moral Ruiz}

${ }^{a}$ Universidad de Jaén, Desp. 224, Edif. C5 Campus Las Lagunillas s/n, 23071 Jaén. lluque@ujaen.es, 'bniversidad de Huelva, Avenida de las Fuerzas Armadas s/n, 21007 Huelva. maria.moral@ddi.uhu.es.

\begin{abstract}
Resumen
El graffiti, surge y se desarrolla como una expresión artística en los muros y el patrimonio mueble de las ciudades, de manera espontánea y por tanto ilegal. Desde entonces, su evolución ha conllevado modificaciones tanto en su consideración social, académica e institucional, como técnica. En la actualidad, no sólo contamos con el graffiti como expresión artística en la calle, sino con todo lo que se ha venido a llamar arte urbano, además del arte público, cuya diferencia fundamental es el carácter de legal o encargado. No obstante, no existe consenso ni en la nomenclatura ni en su génesis. En cualquier caso, ninguna de estas manifestaciones cuentan con una figura de protección. Es cierto que es un arte con carácter efimero, pero la realidad es que nos encontramos ante obras que la propia sociedad pide preservar. Por ello, se reflexionará sobre las posibles fórmulas de catalogación y protección aplicables al arte urbano y el arte público, atendiendo incluso a si debe haber diferencias entre ellos, podrían evitarse asi hechos como arranques sin permiso de los autores. Se usará para ello además el método de la encuesta.
\end{abstract}

Palabras clave: arte urbano, arte público, conservación, difusión, patrimonio inmaterial, material.

\begin{abstract}
Graffiti emerges and develops as an artistic expression in cities' walls and movable heritage in a spontaneous way and as a consequence illegal. Since then, its evolution has led modifications both in its social and academic consideration as a technique. Nowadays, not only graffiti is an artistic expression in the street, but also urban art and related with it, public art which has a commissioned and legal nature. However, there is no consensus in the nomenclature or its genesis. In any case, none of them have any kind of protection. It is true that it is an ephemeral art but the reality is that we are faced with works that the society itself asks to preserve. Therefore, we will reflect on the possible formulas of cataloging and protection to urban art and public art, attending even if there should be differences between them. They could thus avoid facts like pulls without author permission. For that purpose, the survey method will be used.
\end{abstract}

Keywords: urban art, public art, conservation, dissemination, intangible heritage, material. 


\section{Introducción}

La conciencia tutelar comienza a hacerse realmente patente a partir del siglo XVIII, especialmente en Roma, si bien, las sociedades, desde la Antigüedad, aunque se hayan asentado unas sobre otras, destruyendo lo precedente, siempre han sentido la necesidad de conservar ciertas obras, pues, aunque cambiándoles su significación, se apreciaban por su belleza estética o técnica. Eso explica que obras como el Coliseo en Roma, Santa Sofía en Estambul, o en Andalucía la Mezquita de Córdoba y la Alhambra de Granada, hayan persistido a lo largo del tiempo, modificando sus funciones, pero siempre presentes, como testigos de un pasado que dejó un legado que merece ser transmitido a las generaciones futuras, aunque ya no se entiendan sus epigrafías, sus motivos iconográficos o su estructura edilicia. La Ilustración promovió la salvaguarda del patrimonio, pero desde su culto al pasado clásico, a través de las Academias. Es en este mismo momento cuando empiezan a realizarse los primeros catálogos o inventarios, entendidos como el primer paso para el conocimiento y la protección del patrimonio. Pero no será hasta las destrucciones masivas de patrimonio monumental provocado por las Guerras Mundiales, sobre todo la Segunda y la Guerra Civil en España, cuando se tome auténtica conciencia de la importancia de salvaguardar el patrimonio, lo que conllevó la aparición de organismos internacionales como la UNESCO.

En España, la Ley de Patrimonio de 1985, aún vigente, reguló las categorías patrimoniales y los medios de conservación y promoción del patrimonio cultural nacional. Posteriormente, las legislaciones autonómicas desarrollaron más y mejor, en algunos casos como el andaluz, las medidas a tomar. En el caso de Andalucía, es la Ley de 2007 (ahora en proceso de modificación), la que continúa vigente.

La catalogación se convierte así en el instrumento clave para el conocimiento, la protección y la difusión del patrimonio. En este sentido, las legislaciones no sólo prevén la catalogación, sino que esta se hace en base a las distintas categorías de patrimonio, que se resumen en tres grandes grupos dentro del Patrimonio Cultural: Patrimonio Mueble, Patrimonio Inmueble y Patrimonio Inmaterial. Dentro de cada categoría, aparecen subcategorías que permiten ordenar aún más las tipologías patrimoniales. No obstante, la creación artística y las sociedades siempre van por delante de las leyes que las regulan, de manera que las normativas quedan rápidamente obsoletas o simplemente no recogen alguna realidad artística, incluso aunque esté ya asentada. En este texto, se abordará por tanto la situación del arte urbano, sus posibilidades y conflictos, cuestionando cuál es la mejor forma de proteger no ya las piezas, sino la propia creación.

\section{Arte Urbano. Estado de la cuestión}

El concepto de arte urbano, que el grupo de trabajo de Arte Urbano del Grupo Español del International Institute for Conservation of Historic and Artistic Works (GE-IIC), ha definido como "parte de una manifestación artística independiente que se produce en el espacio público, siendo uno de sus principales objetivos convertir el espacio público en un lugar para la experiencia artística, buscando la comunicación directa y apropiándose de elementos del espacio público (...)" (García et al., 2016), tiene una concepción más amplia que el graffiti, si bien esta sea la manifestación urbana más habitual. Queda definido por ser una tipología que se da en el espacio urbano, que es espontánea, efímera y que utiliza como soporte los muros, el mobiliario o los espacios de la ciudad. Sin embargo, a pesar de este carácter en principio vandálico, el arte urbano desde hace algunos años ha comenzado a tener una auténtica consideración por parte de las instituciones con la creación de museos, rutas turísticas, festivales o concursos, donde se fomenta la creación artística urbana, al mismo tiempo que se controla e incluso se aleja a las creaciones de lo urbano, es decir, de su razón de ser. Además el mercado del arte ha comenzado a dar cabida a estas manifestaciones y algunos graffitis arrancados se están vendiendo en las casas de subasta por precios nada desdeñables. No obstante, todo esto se hace sin que exista una legislación que regule este tipo de obras, por lo que la conservación y gestión del arte urbano es aún un tema a debate y un reto.

Por otro lado, socialmente, el arte urbano ha comenzado a tener un alto grado de aceptación y ha llegado a convertirse en promotor de la regeneración de barrios y localidades. Por último, debemos tener en cuenta el muralismo y el arte público, que son obras situadas también en la calle y con características similares, pero realizadas por encargo (García, 2019b). Oscar García señala cómo ahora el juego se ha convertido en una experiencia artística que crea un vínculo entre artista y ciudadano (García, 2019a), pero nos preguntamos si ¿está dejando de ser libre este vínculo? 
A la hora de abordar el marco normativo que envuelve al arte urbano, tenemos que tener en cuenta tres cuestiones. En primer lugar, su definición de vandalismo por las normativas municipales, en segundo lugar, los derechos de propiedad de la obra y en tercer lugar su consideración como patrimonio histórico-artístico.

En relación a lo primero, estas acciones se mueven entre la condena penal y la sanción administrativa. La Comisión de Economía, Fomento; Micro, Pequeña y Mediana Emp; Protec. Consumidores y Turismo de la Cámara, elaboró para la Biblioteca del Congreso Nacional de Chile, un Informe sobre Legislación comparada sobre el delito de Graffiti (2018), en el que comparaba varios países americanos y europeos, de dicho informe se desprende que en todos los países está penado, aunque con distintos tipos de sanciones y agravantes. En España, el Artículo 263 del Código Penal (LO 10/1995) recoge las posibles condenas por daños, aunque no se menciona al graffiti, algunas sentencias lo han usado para establecer condenas sobre todo cuando las pintadas han sido de gran envergadura, por ejemplo, en trenes. Sin embargo, en otros casos el graffiti ha sido penado como falta leve, por deslucimiento, sanción que desde 2015 está recogida en el Artículo 37.13 de la Ley 4/2015 de Protección de la Seguridad Ciudadana (LO 4/2015).

Tenemos que remitirnos a las normativas municipales para encontrar referencias expresas al graffiti. Lo contradictorio de estas normativas en relación a la gestión, es esa dualidad entre la eliminación y el fomento, que hace que los municipios se conviertan en gestores del arte urbano.

En cuanto a la cuestión del derecho de propiedad de la obra, se produce un conflicto entre tres partes, puesto que el muro puede ser parte de una propiedad privada, pero a su vez las fachadas de los inmuebles están sujetas a las normativas municipales, por lo que ya entran dos agentes en juego. No obstante, la propiedad intelectual de la obra es sin duda del autor, siendo además un derecho irrenunciable. Las leyes de propiedad intelectual difieren en cada país, pero Europa reconoce derechos patrimoniales en términos de explotación y compensación económica y derechos morales, irrenunciables, incluso si la obra es ilegal (Giner, 2016).

Por último, en este capítulo debemos tener en cuenta que la legislación sobre protección de patrimonio histórico, ni la estatal ni las autonómicas, contemplan estas manifestaciones, que aún no han sido reconocidas ni incluidas. No obstante, el Instituto Andaluz de Patrimonio Histórico, en su base de datos de patrimonio mueble, ha recogido ya algunos graffitis, lo que les da una legitimización de la que antes carecían y que provoca asimismo su mercantilización. Cabría preguntarse en cualquier caso, si es adecuado que se cataloguen como patrimonio mueble, pues la pintura mural es parte de los inmuebles. Por ejemplo, en Francia, existe el mismo debate sobre si se tratan de obras inseparables del inmueble o si son obras muebles. Goffaux, Guèvel y Seube distinguen entre ellas según el soporte en que se hayan ejecutado y esto influye en la legislación que sanciona las obras ilegales (Goffaux, Guèvel y Seube, 2017). En este sentido, el debate actual se centra en gran medida en si considerar al arte urbano como patrimonio arquitectónico, pictórico, efímero o incluso inmaterial.

El arte urbano, en su diversidad, ha originado la aparición de la subvertiente que supone el arte público, que no es sino un arte urbano por encargo, con una institución generalmente detrás. Esta aparente nimiedad, supone todo un cambio en la concepción del graffiti y el arte urbano, ilegal, subversivo, libre, lo que genera un reto para los investigadores, en cuanto al estudio, conservación e incluso difusión del arte urbano, que con frecuencia se confunde con el arte público. Pero incluso si tratamos sobre el arte público y el muralismo, debemos también cuestionar las formas en que podrían categorizarse, si es que se debiera hacer.

\section{Categorización patrimonial del arte urbano}

¿Debe el arte urbano entrar en alguna categoría patrimonial? En estos momentos, como ya se ha expuesto, ni el arte urbano/graffiti ni el arte público/muralismo, están contemplados en ninguna categoría patrimonial ni en ninguna legislación. Aunque existen excepciones, como la obra dedicada al Músico Jesús Arias (JIP, 2017) del artista granadino conocido como el Niño de las Pinturas, un graffiti situado en el barrio de la Chana que fue declarado como BIC. Este hecho tiene distintas lecturas y supone tanto beneficios como contrapartidas en cuanto a la creación artística ligada al espacio urbano. 
Por un lado, legislar en positivo sobre el arte urbano (es decir, dejando de lado todas las normativas municipales que lo tratan como vandalismo y lo hacen punible), supondría terminar con la propia idiosincrasia del arte urbano en general y del graffiti en particular. De otra parte, al no existir una reglamentación, se producen acciones contra las obras que debieran ser sancionadas, y que sin embargo, se están permitiendo. Con esto no nos referimos a que sobre un graffiti se realice un tag, sino a los arranques y ventas de obras de arte urbano sin consentimiento de los propios autores, o incluso a su musealización.

Además, en caso de incluirlo dentro de una categoría patrimonial, ¿cuál sería? Si atendemos a su pariente más cercano, la pintura mural tradicional, encontramos que si bien está considerada patrimonio inmueble, puesto que forma parte de la piel del edificio y es indesligable del mismo, en numerosos inventarios y catálogos institucionales, aparecen como patrimonio mueble, como ya se ha mencionado.

Algunos autores han usado la fórmula del BiComún, una categoría mancomunada de protección patrimonial que aúna Bien de Interés Cultural y Procomún. El término fue acuñado por la asociación cultural Niquelarte en 2010 (Niquelarte, 2019) y se refiere a los bienes aceptados por una comunidad que no se incluyen dentro de lo legislado en la ley de Patrimonio Histórico Artístico, ni a lo descrito para los Bienes de Interés Cultural (BIC), por lo que serían considerados patrimonio cultural popular. Esta categoría no tiene implicaciones legales, pero permitiría solicitar su protección administrativa para conservarlas y, en su caso, restaurarlas, a través de una iniciativa popular. Esta terminología ha sido usada en el Código Deontológico del Grupo de Arte Urbano del GE-IIC. Por ejemplo, esta categoría se utilizó para salvar la firma de Muelle en la calle Montera de Madrid. Por otro lado, autores como Talego (2012), han considerado al arte urbano como patrimonio inmaterial. El arte urbano puede generar identidad a través de distintos procesos y medios, genera sentimiento de cohesión social a través del reconocimiento, aunque también el debate y muchas críticas (Luque, 2019).

Por otro lado, debe tener en cuenta las diferencias con el arte público (aquel realizado por encargo), puesto que tal vez, debieran tener categorías diferentes. Lo que se haga con el arte público influirá en el arte urbano, ya que están ligados, y deben tener por tanto un tratamiento similar. Por otro lado, se podrían desvincular definitivamente, aunque esto parece más complejo puesto que muchos artistas producen en los dos tipos. Además, muchos profesionales no los diferencian tan claramente o los dividen por otras cuestiones no relativas a la legalidad/ilegalidad con que se ha ejecutado la obra.

\section{Reflexiones de los agentes del arte urbano y el patrimonio}

Puesto que uno de los grandes problemas en cuanto al tema del arte urbano y cómo tratarlo es la falta de consenso, se ha realizado una encuesta a través de google formularios a distintos profesionales: artistas, gestores culturales, conservadores-restauradores, historiadores del arte, periodistas y arquitectos, muchos de ellos, en torno al $61 \%$, especialmente vinculados con el arte urbano/arte público ${ }^{i}$.

La primera cuestión de la encuesta iba encaminada a saber si, en el caso de que el arte urbano fuese reconocido por la legislación con una categoría patrimonial, cuál debería ser. Se daba a escoger entre material, como otras pinturas murales tradicionales; material, con una fórmula nueva; inmaterial o, por último, si no debiera catalogarse en ningún caso. Además, se dejaba abierto el formulario para que pudieran añadirse otras opciones. Si bien las expectativas, eran que la mayoría respondiese que no debería catalogarse, esta opción apenas ha obtenido un $4 \%$. La opción prioritaria (45,5\%), ha sido que se reconozca como bien material con una fórmula nueva, seguido de inmaterial con un (22,7\%). En quienes decidieron incluir otra respuesta, destaca cómo varios señalan que debiera ser una categoría que aúne lo material y lo inmaterial, mientras otros explican que sólo debiera ser documentado, pero no regulado ni intervenido, ya que ha de ser efímero; también se recuerda su ilegalidad; y por último, hay quien destaca que debe atenderse a cada caso. Si diferenciamos entre las respuestas emitidas por aquellos que tienen relación con el arte urbano y los que no, no aparecen diferencias significativas, en ambos grupos gana la opción de "categoría material nueva", relacionada con lo inmaterial, que es la siguiente opción mayoritaria. En concreto, hay quien indica que: "Divulgación y Reconocimiento comunitario, material e inmaterial, de procesos y realidades/contextos (intervención de actores y agentes del barrio)"; "Debe catalogarse tanto como bien material como bien inmaterial, basta recordar que el graffiti o el muralismo urbano son medios de comunicación visual que rescatan los valores y creencias de una comunidad o de un sector de la misma, 
en sus inicios el graffiti nace de la crítica social así como de la búsqueda de identidad de comunidades, el arte urbano es una forma de culturizar a la sociedad en todas sus esferas, ya no es solo por el arte mismo sino por la raíz del arte que es provocar emociones y sentimientos en el caso del arte urbano es crear conciencia de lo que se vive hoy en día y de lo que son nuestras raíces".

En general, aunando estas respuestas, podríamos llegar a la conclusión de que no existe una categoría patrimonial que permita incluir al arte urbano, y que debiera pensarse una nueva en la que lo material y lo inmaterial tengan el mismo peso. Una categoría que proteja de distinta forma, sólo documentando, exceptuando algunos casos concretos cuya vida, por su mayor significación, quizá deba ser alargada en el tiempo. Esa posible categoría patrimonial nueva, debería quedar bien definida para que no incidiese negativamente en la creación artística y sin duda, no sería una categoría legal, sino consensuada por diversos agentes del arte urbano, de manera que todos los agentes involucrados pudieran expresarse y extenderse para actuar en consecuencia.

Preguntados posteriormente por la posibilidad del BIComún, casi a la mitad de los encuestados, les parece que sólo es adecuado en algunos casos concretos donde las obras sean muy significativas, si bien a un 36,4\% les parece una categoría adecuada siempre. A un 9\% les parece una categoría inadecuada siempre, y una respuesta se encaminó a indicar que esta figura sólo es adecuada como un primer paso. Si unimos estas respuestas con las anteriores, podríamos pensar en una categoría novedosa entre lo material y lo inmaterial, que sirva sólo como paso para la documentación, y que además implique una protección mayor cuando así lo decidan las comunidades, promoviendo la participación colectiva. En este caso, sí se aprecian diferencias, aunque no especialmente significativas, entre quienes se dedican a lo urbano y quienes no, pues en el primer grupo gana la opción de que la figura del BIComún es adecuada siempre, seguida de que sólo en algunos casos, mientras que en el grupo de aquellos que no tienen vinculación con el arte urbano, gana la opción de que sólo es adecuada a veces.

Esto, además, se relaciona con la siguiente cuestión planteada, ¿quién o quiénes deben decidir sobre la protección del arte urbano? Los encuestados podían señalar tantas opciones como creyesen convenientes entre: artistas, las comunidades, los profesionales del patrimonio, las instituciones culturales, y las instituciones públicas. Nuevamente se dejaba la opción de incluir nuevas respuestas. La opción "los artistas", recibió 17 votos de 23, seguido de "las comunidades" con 16, las instituciones culturales con 15 y los profesionales del patrimonio con 14. La opción "instituciones públicas", apenas recibió un voto. El espacio libre sirvió en varios casos para indicar que deben ser todos estos elementos unidos quienes tomen las decisiones. Sólo en un caso se añadió la opción "comisarios y críticos de arte", con un sólo voto. Es decir, que si decidiésemos crear esa nueva categoría patrimonial, deberían constituirse grupos de trabajo donde los artistas, las comunidades, las instituciones culturales y los profesionales del patrimonio, esto es, conservadores, restauradores, historiadores del arte, gestores, etc., tuvieran voz y voto, si bien, en el caso de los artistas vivos, estos debieran tener la última palabra. El hecho de que las instituciones públicas no hayan sido votadas, y que sólo una persona haya propuesto a los comisarios y críticos, indica la necesidad de no politizar y sobre todo no mercantilizar las obras de arte urbano. Entre las personas que se dedican al arte urbano, gana la opción que implica a todos los sectores, y en prácticamente todos los casos se da relevancia a los artistas, las comunidades y las instituciones culturales. Sin embargo, entre quienes no se dedican al arte urbano, hay mucha más disparidad entre las opiniones, no existe consenso, lo que indica que es una cuestión quizá más desconocida.

En este punto, se preguntó, dada la diferencia existente entre arte urbano y arte público, aunque a veces se confunden, si debieran tener la misma categoría o distinta. En este caso, se dejaron las respuestas abiertas. La mayoría, se declinaron por que tengan distinta categoría patrimonial, sin embargo, algunos indicaron que la misma. Sorprendentemente es la pregunta que más diferencias ha generado entre las respuestas de quienes se dedican a lo urbano y quienes no, pero con un resultado asombroso, pues los artistas y profesionales dedicados al arte urbano, indican mayoritariamente que arte urbano y arte público tengan la misma consideración, mientras que quienes no se dedican a ello, señalan que deben tener categorías o consideraciones diversas. Este resultado puede venir motivado porque muchos artistas urbanos también están haciendo arte público, y tal vez ellos mismos no crean que existen diferencias entre sus obras o procesos. Pero se contemplan notables diferencias en el hecho de que las obras sean encargadas, ya que son remuneradas y de alguna forma controladas, al indicar el espacio a trabajar, fechas, etc. con respecto a las obras que surgen de forma espontánea, cuándo, dónde y cómo el artista decide. 
Uno de los encuestados indica en relación a esa diferenciación que: "Distintas e iguales. Depende si entendemos el arte urbano como espacio que reflexiona sobre lo urbano (lefebvre) y al arte público como concepto que no se reduce a lo que parece ser arte de rotonda o boulevar". Es interesante que se cite a Lefebvre, que se ocupó de definir el espacio de la ciudad capitalista y se preocupó especialmente por la homogeneización de las ciudades (Lefebvre, 1972). Sin duda, el arte urbano, entendido como manifestación artística espontánea, supone un revulsivo ante esa homogeneidad urbana, mientras que el arte público puede por el contrario reforzar la tendencia institucional hacia la uniformidad.

También un argumento similar aparece en la siguiente respuesta: "Distintas, ya que el arte público ya tiene una identidad y definición propia que fácilmente podría fagocitar una categoría con la que comparte similitudes, pero no al $100 \%$. Quizás lo más importante es trabajar los matices y características propias de una expresión artística concreta”. Es cierto que se puede producir esa fagotización, de hecho, ya se está produciendo puesto que en muchos contextos se emplea el término arte urbano para referirse al arte público y graffiti en lugar de muralismo. Es interesante cómo se propone trabajar con los matices, que en lo referente al arte urbano, lo aporta precisamente esa parte de arte inmaterial, es decir, su relación con el contexto y el factor antropológico.

Por otro lado, se indica que: "Pienso que uno de los conceptos mas importantes y me repito es lo efímero en el espacio público, el tiempo corre tanto como la creatividad en los espacios y no se puede paralizar". Aquí entraría la cuestión de ¿cuánto dura lo efímero? En este sentido, otra persona encuestada señala que: "En este punto debo señalar que en si el grafiti nace en parte gracias al muralismo pero no por ello deban ir precisamente en la misma categoría, lo cual puede crear controversia, ya que por lo general un mural lleva todo un proceso creativo desde el bocetaje, plantación etc, Existen grafitis o murales hechos con la técnica del grafiti que llevan la misma plantación, pero en su mayoría no llevan el mismo proceso creativo que un mural y ahí es donde radica la diferencia". En este sentido podemos preguntarnos qué es lo que prima a la hora de diferenciar: el proceso creativo, la técnica, el contexto, el contenido, la intención, etc.

Otra respuesta interesante, dice en cuanto a si arte urbano y arte público debieran tener la misma categoría o distinta: "La misma, creo... Están en el mismo museo las pinturas negras de Goya y sus trabajos de encargo para la corte". En este sentido, se explica la respuesta mayoritaria de los artistas dedicados al arte urbano frente a la de otros profesionales que no se dedican a ello (si bien esta respuesta la da una artista no dedicada a lo urbano), pues las obras de un mismo autor, para algunos, no pueden considerarse distintas según si son por encargo o no. Ciertamente las obras de muchos creadores urbanos no son distinguibles, un buen ejemplo son las obras del colectivo Boa Mistura, indistinguibles a simple vistas las encargadas de las espontáneas. Además, a las personas que conviven con las obras no parece importarles tanto este factor, sino que se guían por el criterio del gusto.

Por último, se dejó un espacio para añadir aquello que se creyera necesario aclarar. En este apartado han emergido respuestas interesantes. Por ejemplo, una respuesta proveniente de un historiador que no se dedica al arte urbano, señala las diferencias del muralismo y el graffiti, en cuanto al respeto con el entorno, considerando que el muralismo sí respeta ese entorno mientras que el graffiti, aunque tenga valores estéticos, no lo hace. Esta respuesta es sin duda controvertida, pues en muchos foros se ha debatido precisamente lo contrario, la implicación del graffiti con los contextos, mientras que el muralismo a veces conlleva que un artista llegue de otro lugar y sin conocer dónde va a trabajar deje su obra, provocando que no exista integración en el barrio ni implicación social, y por lo tanto se produzca un rechazo de la comunidad, frente a la identidad que pueden aportar otras obras. Es cierto que actualmente, muchos festivales e iniciativas que producen arte público, promueven la relación artista/sociedad, de forma que las obras están mejor integradas en sus contextos y son bien aceptadas. En realidad, muchas veces la sociedad no distingue entre si es encargado o vandálico, sino simplemente si les gusta o no, como demuestran casos como el "graffiti de las ratas" del Soho de Málaga, por encargo, que horrorizó al vecindario frente a la aparición de unas caras en la muralla de la Puerta de Almodóvar en Córdoba, que mucha gente quiso que dejaran (Luque, 2019).

En otro caso se indica que: "Por mi parte entiendo el arte público/urbano como una manifestación de índole urbana que trata de afrontar cuestiones y problemáticas sociales relativas al espacio físico de la ciudad desde procesos que pueden ser colectivos, participativos o individuales (expresiones idiosincráticas) son actos de acción directa, muy politizados, críticos con la institucionalidad y la heteronormatividad, todo lo contrario a un encargo institucional o corporativo ¿qué sentido tiene que esté protegido desde lo institucional? Le bastaría con un entendimiento y reconocimiento 
comunitario". Esta aportación es interesante ya que va muy en la línea de lo planteado desde el Grupo de Arte Urbano del GE-IIC, ciertamente, parece que una protección legal desvirtuaría completamente lo que es el arte urbano, su propia génesis, su razón de ser, esto no implica, que desde las instituciones culturales o los profesionales del sector, no se haga nada en relación a esto, sino que es posible trabajar en una línea pedagógica, es decir, ayudar a comprender una manifestación artística y cultural, en pro de conseguir un reconocimiento como tal por parte de las comunidades y no tanto de las instituciones.

En este sentido también hay quien indica que: "El muralismo/graffiti tiene un componente clandestino y alegal que hace que quizá se desnaturalice al ser reconocido", y en otro caso se señala que: "el arte urbano es ilegal y solo casos muy concretos se deben conservar. Debería catalogarse lo que hay en la calle estableciendo unos criterios profesionales y comisariado técnico". Pero ¿no ha sido el arte urbano ya reconocido? La imparable aparición no ya de rutas, festivales y demás, sino de museos de arte urbano, que están exhibiendo obras o bien hechas expresamente en el interior del museo (que hay que cuestionar como urbanas ya que no surgen en el espacio urbano), obras de artistas urbanos, que no son urbanas, sino serigrafías y otras tipologías, e incluso obras arrancadas sin permiso de los autores, hace que ese reconocimiento ya haya llegado, provocando esa desnaturalización de lo que era en sus orígenes el graffiti y el arte urbano. Vemos arte urbano en la mesa que utiliza el presentador Broncano en su programa, La Resistencia, realizado por Okuda, o a Belin en el videoclip de Infinitos Bailes del cantante Raphael, o en un cartel de semana santa de Málaga. Es esto lo que hace tan necesaria la reflexión en torno a cómo debe ser reconocido para evitar malas praxis, por ejemplo, en su exhibición. Por otro lado, la necesidad de catalogar, o al menos inventariar las obras sí parece clara, desde un punto de vista profesional, que incluya perfiles técnicos como historiadores del arte y conservadores/restauradores. No obstante, la opinión del artista al respecto también debe tenerse en cuenta. Incluso se podría implicar a las comunidades en ello. Por otra parte, las redes sociales, incluso las de los propios artistas, están en cierta medida haciendo esta labor.

\section{5. ¿El arte urbano como patrimonio inmaterial?}

Debemos entender por tanto, atendiendo a los autores mencionados y a las encuestas realizadas, que el arte urbano necesita definirse con una categoría que nos permita emplear a todos un mismo lenguaje y que asegure una buena praxis en cuanto a su estudio y exposición. No obstante, no se trataría de una categoría dentro del marco de la legislación patrimonial que pudiera perjudicar la propia práctica artística. Dicha categoría, debiera estar a medio camino entre lo material y lo inmaterial, con especial importancia de este segundo aspecto, entendiendo lo inmaterial como un patrimonio vivo, de transmisión oral, que fomenta la diversidad cultural frente a la globalización (UNESCO, 2019) y que proporciona identidad a las sociedades, fomentando la cohesión y la integración y que se convierte en algo representativo de las comunidades. La UNESCO, además incluye dentro del patrimonio inmaterial usos urbanos contemporáneos. Según el organismo internacional, "el patrimonio cultural inmaterial sólo puede serlo si es reconocido como tal por las comunidades" (UNESCO, 2019), y parece que queda claro que el arte urbano ha sido ya reconocido. No obstante, debe diferenciarse entre arte urbano y público y preguntarse si hay que catalogarlo de la misma forma, con la misma figura. Probablemente, sería positivo entenderlos como dos subcategorías dentro de un mismo grupo, pues ambos conviven y están estrechamente relacionados.

Dicha categoría patrimonial, debiera asegurar que el arte urbano no perdiese su génesis, es decir, no puede intervenir en los modos, en la propia creación, sino simplemente servir para estudiar los procesos, inventariar los resultados materiales y difundir el conocimiento sin incidir en la propia manifestación artística. Es decir, los museos de arte urbano que están surgiendo, deberían, por ejemplo, aclarar su nomenclatura, no deberían exponer obras arrancadas o sin permiso de los autores. Estos museos deberían simplemente divulgar la existencia del arte urbano y desentrañar parte de sus significados para hacerlos accesibles al público, de manera que no se convirtiera el arte urbano en un objeto descontextualizado y mercantilizado, en un bien de consumo, como otro tipo de manifestaciones artísticas contemporáneas. Debe subrayarse en este caso la importancia de la participación activa de las colectividades. Esta nueva categoría no debiera tampoco tomar de lo etnológico las medidas de fomento para la conservación de la práctica, porque sería otra forma de institucionalizar e incidir en ella de forma negativa. El arte urbano debe desarrollarse por sí mismo, de forma independiente, sin que los profesionales del patrimonio podamos más que estudiarlo. 
Lo describía de forma muy acertada uno de los encuestados especificando que el arte urbano es un: "Bien inmaterial. Reconocimiento de procesos y contextos pero no conservación ni protección de obras. El arte urbano es presente. Su valor de ocupación de espacios públicos y socialización está por encima de resultados concretos. Apuesto por no la intervención desde fuera de la comunidad".

\section{Conclusiones}

En conclusión, el arte urbano está sin duda sujeto aun a debate en muchos aspectos, como la propia definición y nomenclatura de los distintos fenómenos vinculados a él. Se debe diferenciar arte urbano y público, pero teniendo en cuenta que están ligados y que los propios artistas, como revela la encuesta realizada, no los diferencian tanto por el hecho de ser espontáneos o arrancados. Parece claro que no debe protegerse ni categorizarse desde la legislación, pero sí debe buscarse una forma de entenderlo que permita la buena praxis, una nueva forma, cercana al patrimonio inmaterial, en la que se tengan más en cuenta los procesos y los contextos, además de la opinión de cada artista, siendo para ello la encuesta y la entrevista una herramienta de trabajo fundamental, así como es fundamental la implicación de la gente, no sólo del ámbito académico. No obstante, sería preciso ampliar más la encuesta para tener resultados más significativos.

\section{Referencias}

García García, O. (2019a). Dios salve al arte contemporáneo. España: Paidós.

García Gayo, E. (2019b). El espacio intermedio del arte urbano. Ge-Conservación, 16, 154-165. Recuperado de https://geiic.com/ojs/index.php/revista/article/view/704/917

García Gayo, E. et al. (2016). Anexo I: Propuesta de código deontológico para la conservación y restauración de arte urbano. GeConservación, 10, 186-192. Recuperado de https://ge-iic.com/ojs/index.php/revista/article/view/419

Giner Cordero, E. (2016). Propiedad intelectual y arte urbano. Mural Street Art Conservation, 3, 28-31.

Goffaux Callebaut, G., Guével, D., y Seube, J. B. (2017). Droit(s) et Street Art. De la transgression à l'artification. Francia: LGDJ.

JIP. (2017, 6 de mayo). El Ayuntamiento de Granada declara de Interés Cultural el mural dedicado a Jesús Arias en la Chana, del Niño de las Pinturas, El Independiente de Granada, s.p. Recuperado de http://www.elindependientedegranada.es/ $\underline{\text { cultura/ayuntamiento-granada-declara-interes-cultural-mural-dedicado-jesus-arias-chana-nino-pinturas }}$

Lefebvre, H. (1972). La revolución urbana. España: Alianza

Luque Rodrigo, L. (2019). Identidad e imagen de la ciudad contemporánea: los museos de arte urbano. Ge-Conservación, 16, 176185. Recuperado de https://ge-iic.com/ojs/index.php/revista/article/view/706/919

Meraviglia, M. (2017). Street art e diritto: analisi comparatistica della tutela autoriale negli Stati Uniti ed in Italia. Tesi di Laurea. Lugano: SUPSI.

Niquelarte (2013). BIComun ThinkCommons (03) \#BICommons. España. Recuperado de http://niquelarte.bicomun.org/es/bicomunthinkcommons-03-bicommons-2/

Quirosa García, M. V. (2005). Historia de la protección de los bienes culturales muebles: definición, tipologías y principios generales de su estatuto jurídico. Granada: Universidad de Granada.

Talego, F. (2012). Grafitis: huellas de un patrimonio inmaterial urbano. Mus-A: Revista de los museos de Andalucía, 13 , 36-37.

UNESCO. (2019). ¿Qué es el patrimonio cultural inmaterial?. España. Recuperado de https://ich.unesco.org/es/que-es-elpatrimonio-inmaterial-00003

\footnotetext{
${ }^{\mathrm{i}}$ Las personas que han participado en la encuesta son: José Luis Linares Robles; Dora I Ferreira Burgher; Basurama; Pablo; Diego Ortega Alonso; Elena García Gayo; V; Eduardo D’Acosta; Ygor Marotta; María Ortega; Gabriela Martínez Alarcón; Ana Quesada Cerezo; Matias Mata (Sabotaje al Montaje); Santiago; Fran Pérez Rus; Vermibus; Icat (Mónica Gómez Martínez); Dadospuntocero; Lourdes. Agradecemos su participación.
} 